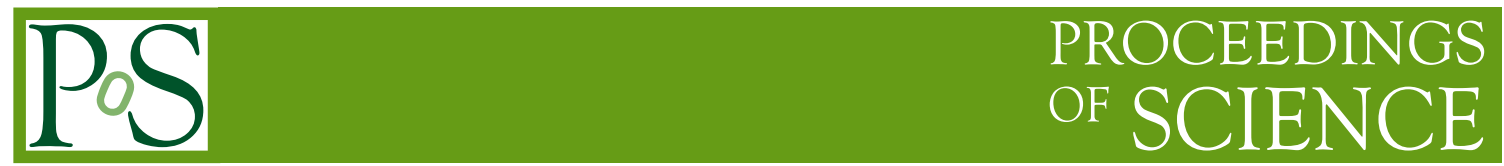

\title{
European Particle Physics Strategy Update
}

\author{
Brigitte Vachon* \\ McGill University \\ E-mail: Brigitte.Vachon@McGill.ca
}

The European Particle Physics Strategy update process and its associated timeline are presented. As part of this process, a CERN Council Open Symposium was held on 13-16 May 2019, in Granada, Spain. Selected highlights from the symposium are summarized as well as general considerations that emerged from discussions that took place at the symposium. Finally, the next steps in the process to update the European Strategy for Particle Physics are presented.

XXIX International Symposium on Lepton Photon Interactions at High Energies - LeptonPhoton2019 August 5-10, 2019

Toronto, Canada

${ }^{*}$ Speaker. 


\section{Introduction}

The European Particle Physics Strategy Update (EPPSU) is the process through which the European community reviews and updates the priorities and strategy of the field of particle physics [1]. The process, involving a broad community consultation, culminates into a strategy driven by sicentific questions but also taking into account technical and financial feasibility.

The first such European Particle Physics Strategy was initiated by CERN Council in 2005 and concluded in 2006 [2]. An update to this strategy was later endorsed by CERN Council in 2013 [3].

The process to prepare a second update to this strategy was launched by the CERN Council in September 2018 [4]. This new Strategy Update, taking into account the global nature of the field and developments in related fields, will guide the direction of particle physics into the mid-2020s and beyond.

\section{EPPSU Process}

The overall strategy update process is coordinated by the Strategy Update Secretariat (SUS) [5]. The strategy document is drafted by the European Strategy Group (ESG) [6]. The ESG is assisted in this task by the Physics Preparatory Group (PPG) [7]. The PPG is charged with preparing the scientific contribution to the work of the ESG, in a so-called "Briefing Book" document. The strategy update is finally adopted by the CERN Council and the CERN Director-General is responsible for its implementation.

Figure 1 summarizes the process and timeline of key events for the update of the European Particle Physics Strategy. The process was officially launched in September 2018. An Open Symposium was organized and held in Granada, Spain, in May 2019. The actual strategy will be drafted in January 2020, in time to be submitted to and approved by the CERN council in May 2020.

In response to a call for community inputs issued in February 2018, a total of 160 written submissions were received. These submissions were processed by the PPG and organized into 11 different categories. The scientific program of the CERN Council Open Symposium on the update of the European Strategy for Particle Physics was then developed based on these written inputs.

\section{Open Symposium Highlights}

The CERN Council Open Symposium on the update of the European Strategy for Particle Physics [8] was held on 13-16 May 2019, in Granada, Spain. The symposium consisted of both parallel and plenary sessions, organized and convened by members of the PPG. Parallel sessions were organized around 8 different themes: Accelerator science and technology, Instrumentation and computing, Electroweak physics, Strong interactions, Neutrino physics, Beyond Standard Model (BSM) at colliders, Dark matter and dark sector, Flavour physics and CP violation. The plenary sessions contained presentations on the current status of the field, summaries of inputs from different communities, and summaries from parallel sessions. The format of the symposium was designed to provide sufficient time for detailed discussions to take place. A total of 603 participants attended the symposium, $12 \%$ of whom came from outside Europe. 


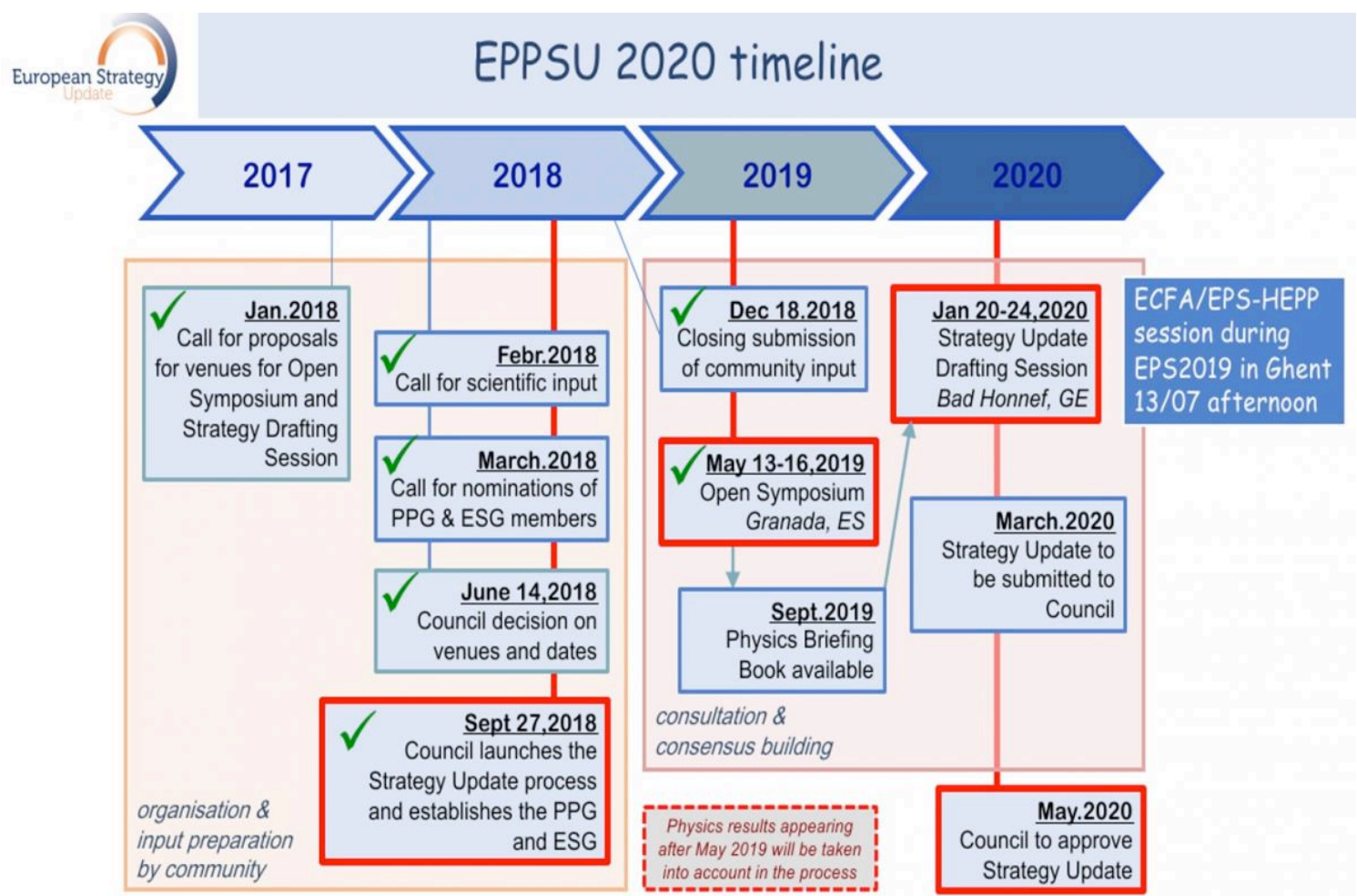

Figure 1: Diagram summarizing the process and timeline of key events in the 2020 European Particle Physics Strategy Update [9].

While it is impossible to summarize the wealth of information that was presented and discussed at the symposium, the following paragraphs present a few selected highlights chosen by the author. The interested reader is encouraged to directly review all the documents presented at the symposium that can be accessed directly from the symposium agenda page [8].

On the first day of the symposium, a status update of the implementation of the 2013 European Particle Physics Strategy Update was presented by the CERN Director-General [10]. Some of the notable accomplishments to date reported included, but are not limited to,

- the completion of the LHC Run-2 data taking campaign, with accelerators, detectors and computing performance exceeding expectations;

- the approval of the High Luminosity LHC (HL-LHC) project by CERN Council in June 2016;

- accelerator R\&D work and design studies such as those carried out for next generation superconducting magnets, CLIC, FCC, and AWAKE;

- the establishment of the CERN neutrino platform in 2014;

- the setting up of a Physics Beyond Colliders Study group in 2016, charged to explore compelling projects complementary to high-energy colliders; 
- the start of a new project, in 2018, for the education of the general public: the Science Gateway project.

Perspectives on the European Strategy Update from communities in the Americas [11] and Asia [12] were also presented.

From a physics point of view, it was made clear that this is a very interesting yet puzzling time for particle physics. The properties of the Higgs boson are so far compatible with those expected from the Standard Model (SM), neutrinos have masses not acquired within the strict confines of the SM, there are no (additional) signs of the existence of new physics phenomena beyond the SM in the various particle physics experimental projects, there is compelling evidence for the existence of dark matter in the universe with no candidate particle(s) in the SM, and there is an observed prevalence of matter over anti-matter in the universe. To address these puzzles, the particle physics community is engaged in a rich and diverse research program, as presented at the symposium.

This research program includes the detailed study of strong interactions in different energy regimes spanning several orders of magnitude. These studies aim to understand, for example, the properties of hadrons (e.g. proton radius, proton inner structure), how properties of the quark-gluon plasma (QGP) emerge from the fundamental interactions of quantum chromo-dynamics (QCD), as well as include precision tests of QCD predictions. Furthermore, the experimental knowledge and theoretical understanding of QCD is vital to succeed in the exploration of nature in other areas of research such as the study of neutrino properties, the electroweak sector, flavour physics and searches for BSM.

The outstanding sensitivity to BSM physics processes in the flavour sector was presented, as well as the strong synergy that exists between low-energy precision and high-energy frontier experiments in flavour physics. The coming years will be very interesting for flavour physics, as several physics programs come into full swing and new experiments are being designed.

The study of neutrinos provides a complementary window to search for BSM physics. A diverse experimental program focused on understanding the properties of these elusive particles was presented, with the goal of ultimately understanding what may be the new physics mass scale.

Beyond neutrino physics, it is clear that there is a strong physics case for multi-messenger physics, with a high impact on particle physics. One example of this is in the exploration of the dark side of the universe. Here, particle physics experiments are desiged to cover an extremely wide ranges of energy scales, using a variety of different experimental techniques. Discussions at the symposium pointed to an emerging consensus on the need for more coordination between accelerator based, direct detection and indirect detection dark sector searches, for the common interpretation of results. Better coordination will also be of fundamental importance to validate, through different channels, a possible dark matter discovery.

Discussions also took place in Granada regarding other important research ingredients necessary to achieve the desired scientific outcomes. One of these ingredients is the detector R\&D work needed to address the technological challenges faced by future experiments such as micron resolution with very low mass, picosecond time resolution, very high radiation tolerance, ability to combine features, high-performance photodetectors, very large area coverage at low cost, etc. Another ingredient enabling our scientific outcomes is the implementation of an effective computing infrastructure. Addressing the computing challenges of future experimental projects will require 
carefully planned and coordinated R\&D efforts on many fronts. Also, it was shown that the need to extract physics in the most efficient way given a fixed amount of resources requires an increasingly "holistic" approach to the design of experiments. For example, the detector design decisions such as geometry or segmentation can either ease or place a huge burden on the offline computing and simulation. Finally, underlying these different research ingredients are the scientists themselves. For the future of the field it is crucial to address human factors such as the training of the next generation of experts, and the successful recruitment and retention of the most talented researchers, by providing, for example, interesting career opportunities for detector and computing experts.

Finally, the assessment and comparison of different proposed future collider projects was also a key topic of discussion at the symposium. Figure 2 shows a summary of the main collider projects discussed, along with some of their characteristics such as center-of-mass energy, integrated luminosity, power and cost. Some of the major technological challenges for each collider project are summarized in Figure 3. These include, for hadron colliders, the development of high-field magnets, and for lepton colliders, the production of high-Q/high gradient superconducting RF cavities. Another challenge for the field of particle physics is the very long timescales involved in the development and construction of the next collider project(s).

\begin{tabular}{|c|c|c|c|c|c|c|}
\hline Project & Type & $\begin{array}{l}\text { Energy } \\
{[\mathrm{TeV}]}\end{array}$ & Int. Lumi. $\left[a^{-1}\right]$ & $\begin{array}{l}\text { Oper. Time } \\
\text { [y] }\end{array}$ & $\begin{array}{l}\text { Power } \\
\text { [MW] }\end{array}$ & Cost \\
\hline \multirow[t]{3}{*}{ ILC } & ee & 0.25 & 2 & 11 & 129 (upgr. 150-200) & 4.8-5.3 GILCU + upgrade \\
\hline & & 0.5 & 4 & 10 & $163(204)$ & 7.98 GILCU \\
\hline & & 1.0 & & & 300 & $?$ \\
\hline \multirow[t]{3}{*}{ CLIC } & ee & 0.38 & 1 & 8 & 168 & $5.9 \mathrm{GCHF}$ \\
\hline & & 1.5 & 2.5 & 7 & (370) & $+5.1 \mathrm{GCHF}$ \\
\hline & & 3 & 5 & 8 & (590) & $+7.3 \mathrm{GCHF}$ \\
\hline \multirow[t]{2}{*}{ CEPC } & ee & $0.091+0.16$ & $16+2.6$ & & 149 & \multirow[t]{2}{*}{$5 \mathrm{G \$}$} \\
\hline & & 0.24 & 5.6 & 7 & 266 & \\
\hline \multirow[t]{3}{*}{ FCC-ee } & ee & $0.091+0.16$ & $15+10$ & $4+1$ & 259 & \multirow[t]{2}{*}{$10.5 \mathrm{GCHF}$} \\
\hline & & 0.24 & 5 & 3 & 282 & \\
\hline & & $0.365(+0.35)$ & $1.5(+0.2)$ & $4(+1)$ & 340 & $+1.1 \mathrm{GCHF}$ \\
\hline LHeC & ep & $60 / 7000$ & 1 & 12 & $(+100)$ & $1.75 \mathrm{GCHF}$ \\
\hline FCC-hh & $\mathrm{pp}$ & 100 & 30 & 25 & $580(550)$ & 17 GCHF (+7 GCHF) \\
\hline FCC-NbTi & $p p$ & 37.5 & 10 & 20 & 240 & 14 GCHF (including tunnel) \\
\hline HE-LHC & $\mathrm{pp}$ & 27 & 20 & 20 & & $7.2 \mathrm{GCHF}$ \\
\hline
\end{tabular}

Figure 2: Table summarizing some of the characteristics of the main collider projects considered by the community [13]. Note that the entry in red was added to this table after the symposium at the request of the ESG, based on discussions that took place during the symposium.

At the symposium, the status of development of future accelerator technologies was also presented. This included presentations on ongoing R\&D work to realize a muon collider and on recent developments in plasma wakefield acceleration. It was shown that, in order to address key challenges of future accelerator techologies, vigorous $R \& D$ in a strongly coordinated global effort is required. 


\begin{tabular}{|c|c|c|c|c|c|c|c|c|c|}
\hline & & Ref. & $\begin{array}{c}\text { E } \\
(\mathrm{CM}) \\
\text { [aV] }\end{array}$ & $\begin{array}{c}\text { Luminos } \\
\text { ity } \\
\text { [1E34] }\end{array}$ & $\begin{array}{l}\text { AC- } \\
\text { Power } \\
\text { [MW] }\end{array}$ & $\begin{array}{c}\text { Cost-estimate } \\
\text { Value* } \\
\text { [Billion] }\end{array}$ & $\begin{array}{c}\mathrm{B} \\
{[\mathrm{T}]}\end{array}$ & $\begin{array}{c}E: \\
{[M V / m]} \\
(\mathrm{GHz})\end{array}$ & Major Challenges in Technology \\
\hline & $\begin{array}{l}F C C- \\
N b T i\end{array}$ & $\begin{array}{l}\text { (to be } \\
\text { filled) }\end{array}$ & $\sim 100$ & $<30$ & & & $\sim 6$ & & ...Find the people who want to do it \\
\hline & $\begin{array}{l}\text { FCC- } \\
\text { hh }\end{array}$ & CDR & $\sim 100$ & $<\mathbf{3 0}$ & 580 & $\begin{array}{l}24 \text { or } \\
+17 \text { (aft. ee) } \\
{[\text { [BCHF] }}\end{array}$ & $\sim 16$ & & $\begin{array}{l}\text { High-field SC magnet (SCM) } \\
\text { - Nb3Sn: Jc and Mechanical stress } \\
\text { Energy management }\end{array}$ \\
\hline hh & SPPC & $\begin{array}{l}\text { (to be } \\
\text { filled) }\end{array}$ & $\begin{array}{l}75- \\
120\end{array}$ & TBD & TBD & TBD & $\begin{array}{c}12- \\
24\end{array}$ & & $\begin{array}{l}\text { High-field SCM } \\
\text {-IBS: Jcc and mech. stress } \\
\text { Energy management }\end{array}$ \\
\hline C & $\begin{array}{l}\text { FCC- } \\
\text { ee }\end{array}$ & CDR & $\begin{array}{l}0.18- \\
0.37\end{array}$ & $\begin{array}{c}460- \\
31\end{array}$ & $\begin{array}{c}260- \\
350\end{array}$ & $\begin{array}{l}10.5+1.1 \\
{[\mathrm{BCHF}]}\end{array}$ & & $\begin{array}{c}10-20 \\
(0.4-0.8)\end{array}$ & $\begin{array}{l}\text { High-Q SRF cavity at }<\mathrm{GHz}, \mathrm{Nb} \text { Thin-film Coating } \\
\text { Synchrotron Radiation constraint } \\
\text { Energy efficiency (RF efficiency) }\end{array}$ \\
\hline C & CEPC & CDR & $\begin{array}{c}0.046- \\
0.24 \\
(0.37)\end{array}$ & $\begin{array}{c}32 \sim \\
5\end{array}$ & $\begin{array}{l}150- \\
270\end{array}$ & $\begin{array}{c}5 \\
{[B \$]}\end{array}$ & & $\begin{array}{c}20-(40) \\
(0.65)\end{array}$ & $\begin{array}{l}\text { High-Q SRF cavity at < GHz, LG Nb-bulk/Thin-film } \\
\text { Synchrotron Radiation constraint } \\
\text { High-precision Low-field magnet }\end{array}$ \\
\hline & ILC & $\begin{array}{l}\text { TDR } \\
\text { update }\end{array}$ & $\begin{array}{l}0.25 \\
(-1)\end{array}$ & $\begin{array}{c}1.35 \\
(-4.9)\end{array}$ & $\begin{array}{c}129 \\
(-300)\end{array}$ & $\begin{array}{l}\text { 4.8- 5.3 } \\
\text { (for } 0.25 \mathrm{TeV} \text { ) } \\
\text { [BILCU] }\end{array}$ & & $\begin{array}{l}31.5- \\
(45) \\
(1.3)\end{array}$ & $\begin{array}{l}\text { High-G and high-Q SRF cavity at GHz, Nb-bulk } \\
\text { Higher-G for future upgrade } \\
\text { Nano-beam stability, e+ source, beam dump }\end{array}$ \\
\hline C & CLIC & CDR & $\begin{array}{l}0.38 \\
(-3)\end{array}$ & $\begin{array}{c}1.5 \\
(-6)\end{array}$ & $\begin{array}{c}160 \\
(-580)\end{array}$ & $\begin{array}{c}\mathbf{5 . 9} \\
\text { (for } 0.38 \mathrm{TeV} \text { ) } \\
\text { [BCHF] }\end{array}$ & & $\begin{array}{c}72-100 \\
\text { (12) }\end{array}$ & $\begin{array}{l}\text { Large-scale production of Acc. Structure } \\
\text { Two-beam acceleration in a prototype scale } \\
\text { Precise alignment and stabilization. timing }\end{array}$ \\
\hline
\end{tabular}

Figure 3: Summary table of some of the major technological challenges of future collider projects considered by the community [14].

A comparison of the physics reach of different proposed future collider projects was also presented $[15,16]$. For example, Figure 4 shows the expected relative precision $(\%)$ of $\kappa$ parameters (Higgs coupling strength modifier parameters) achievable at different future colliders in the study of Higgs interactions with different particles. This comparison was carried out not only for different collider programs but also for different combinations of experiments. Comparisons of the physics reach of different collider projects in BSM searches were also presented [17].

\section{Next steps}

The next step in the European strategy update process is the preparation of the Briefing Book. The Briefing Book will summarize the written inputs submitted by the community and the outcomes of the symposium. This document will be prepared by members of the PPG and is expected to be ready by September $2019^{1}$.

Some of the community feedback and considerations that have been raised to date include

- an emerging consensus for the importance of a "Higgs factory" to fully explore properties of the Higgs boson, electroweak sector, etc;

- the need to prepare a clear path towards achieving the highest energy;

- the need for vigorous $\mathrm{R} \& \mathrm{D}$ in accelerator technologies, detectors and computing for realizing future projects;

\footnotetext{
${ }^{1}$ The Briefing Book [18] is now publically available at the time of publication of these proceedings.
} 
$\kappa_{W}(\%)$
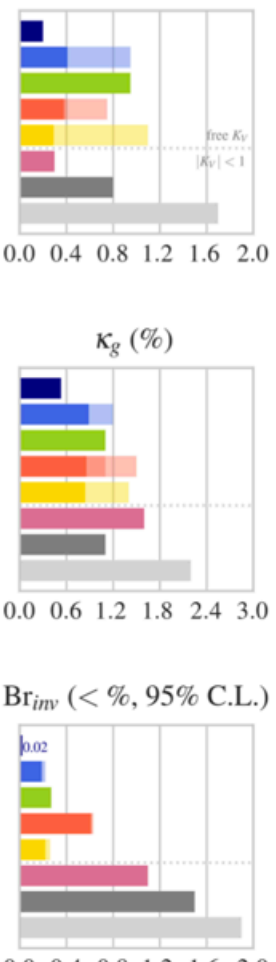

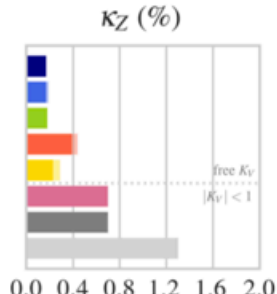

$\kappa_{\gamma}(\%)$

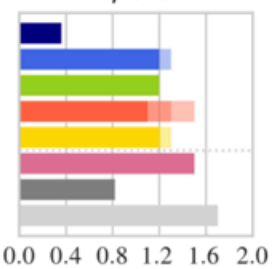

$\begin{array}{llllll}0.0 & 0.4 & 0.8 & 1.2 & 1.6 & 2.0\end{array}$
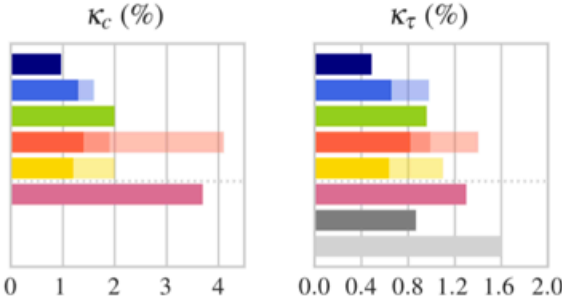

$\begin{array}{llllll}0.0 & 0.4 & 0.8 & 1.2 & 1.6 & 2.0\end{array}$

$\kappa_{t}(\%)$

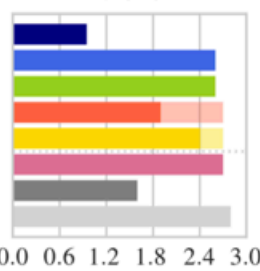

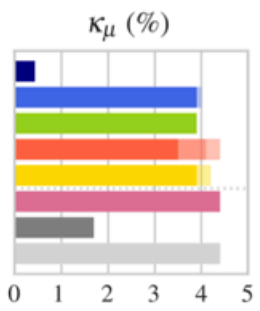

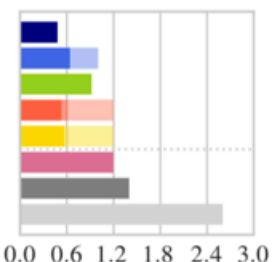

$\kappa_{b}(\%)$

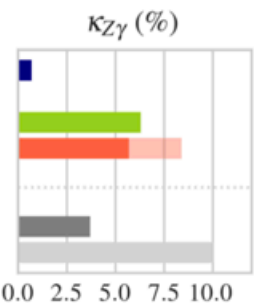

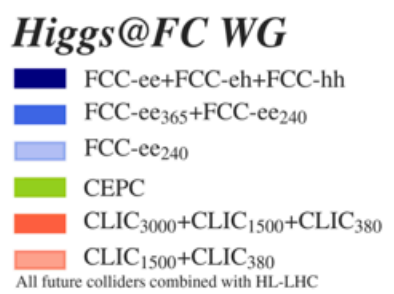

Kappa-3, May 2019

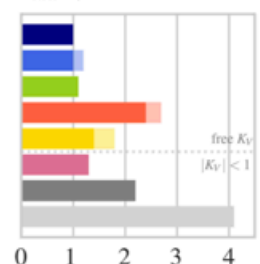

Figure 4: Expected relative precision (\%) of $\kappa$ parameters (Higgs coupling strength modifier parameters) achievable at different future colliders in the study of Higgs interactions with different particle $[15,16]$.

- a strong case for the support of a diverse research program in particle phyics, e.g. complementing the energy frontier with precision measurements, both from a physics point of view as well as to ensure that particle physics remains attractive over the long development time scales of future projects;

- the importance of further exploiting synergies and complementarities between fields;

- the need for adequate support for theory development and tools;

- the importance for the development and preservation of expertise in detector, accelerator and computing areas;

- the importance of communication of particle physics goals and interests to other fields of science and to society;

- the need to consider innovative ways to increase research funding.

\section{Summary}

The process to update the European particle physics strategy is ongoing and will conclude with the approval, by the CERN Council, of an updated strategy, in May 2020. As part of this process, an 
Open Symposium was organized and took place in Granada, Spain in May 2019. Members of the PPG are currently drafting the Briefing Book document to be submitted to the ESG in September $2019^{2}$. The strategy will be drafted by the ESG in January 2020. It is clear that this is a very exciting, yet challeging, time for particle physics.

\section{Acknowledgments}

The author wishes to thank members of the organizing and program committees of the XXIX International Symposium on Lepton Photon Interactions at High Energies for the invitation to present at the meeting and the hosting of a stimulating and fruitful meeting.

The author also wishes to extend her gratitude to members of the Strategy Update Secretariat for their useful suggestions and inputs in the preparation of this presentation.

\section{References}

[1] European Particle Physics Strategy: https:

//council.web.cern. ch/en/content/european-strategy-particle-physics

[2] European Particle Physics Strategy 2006:

http: //council-strategygroup.web.cern.ch/council-strategygroup/

[3] European Particle Physics Strategy Update 2013:

http: //europeanstrategygroup.web.cern. ch/europeanstrategygroup/

[4] European Particle Physics Strategy Update 2020:

https: / / europeanstrategyupdate.web.cern.ch/

[5] Strategy Update Secretariat (SUS) composition:

https://europeanstrategyupdate.web.cern.ch/strategy-secretariat

[6] European Strategy Group (ESG) composition: https :

/ / europeanstrategyupdate.web.cern. ch/european-strategy-group-esg

[7] Physics Preparatory Group (PPG) composition: https :

/ / europeanstrategyupdate.web. cern.ch/physics-preparatory-group-ppg

[8] CERN Council Open Symposium on the Update of the European Strategy for Particle Physics: https://cafpe.ugr.es/eppsu2019/

[9] European Particle Physics Strategy Update 2020 timeline:

https: / / europeanstrategyupdate.web.cern.ch/process-0

[10] F. Gianotti, "Implementation of the 2013 European Strategy Update", presentation at the CERN Council Open Symposium on the update of the European Strategy for Particle Physics, 13-16 May 2019, Granada, Spain.

[11] Y.K. Kim, "Perspective on the European Strategy from the Americas", presentation at the CERN Council Open Symposium on the update of the European Strategy for Particle Physics, 13-16 May 2019, Granada, Spain.

\footnotetext{
${ }^{2}$ The Briefing Book [18] document is now publically available.
} 
[12] G. Taylor, "Perspective on the European Strategy from Asia", presentation at the CERN Council Open Symposium on the update of the European Strategy for Particle Physics, 13-16 May 2019, Granada, Spain.

[13] D. Schulte, "Technical Overview and Challenges of Proposed Higgs Factories", presentation at the CERN Council Open Symposium on the update of the European Strategy for Particle Physics, 13-16 May 2019, Granada, Spain.

[14] A. Yamamoto, "State of the Art and Challenges in Accelerator Technologies - Past and Present", presentation at the CERN Council Open Symposium on the update of the European Strategy for Particle Physics, 13-16 May 2019, Granada, Spain.

[15] M. Cepeda, "Higgs precision measurements at future colliders", presentation at the CERN Council Open Symposium on the update of the European Strategy for Particle Physics, 13-16 May 2019, Granada, Spain.

[16] J. de Blas et al., "Higgs Boson Studies at Future Particle Colliders", arXiv:1905.03764 [hep-ph].

[17] J. A. Maestre, "EWSB dynamics and resonances: experimental focus", presentation at the CERN Council Open Symposium on the update of the European Strategy for Particle Physics, 13-16 May 2019, Granada, Spain.

[18] R.K. Ellis et al., "Physics Briefing Book : Input for the European Strategy for Particle Physics Update 2020”, CERN-ESU-004, arXiv:1910.11775 [hep-ex]. 